PROCEEDINGS OF THE

AMERICAN MATHEMATICAL SOCIETY

Volume 139, Number 7, July 2011, Pages 2463-2472

S 0002-9939(2010)10691-3

Article electronically published on December 3, 2010

\title{
ESTIMATES OF GRADIENT AND OF JACOBIAN OF HARMONIC MAPPINGS DEFINED IN THE UNIT DISK
}

\author{
DAVID KALAJ
}

(Communicated by Mario Bonk)

\begin{abstract}
Let $F: \mathbb{T} \rightarrow \gamma$ be a bounded measurable function of the unit circle $\mathbb{T}$ onto a rectifiable Jordan curve $\gamma$ with the length $|\gamma|$, and let $w=P[F]$ be its harmonic extension to the unit disk $\mathbb{U}$. By using the arc length parametrization of $\gamma$ we obtain the following results: (i) If $F$ is a quasi-homeomorphism and $1 \leq p<2$, the $L^{p}$-norm of the Hilbert-Schmidt norm of the gradient of $w$ is bounded as follows: $\|D(w)\|_{p} \leq \frac{|\gamma|}{4 \sqrt{2}}\left(\frac{16}{\pi(2-p)}\right)^{1 / p}$. (ii) If $F$ is $p$-Lipschitz continuous and $\gamma$ is Dini smooth, then the Jacobian of $w$ is bounded in $\mathbb{U}$ by a constant $C(p, \gamma)$. The first result is an extension of a recent result of Verchota and Iwaniec, and Martin and Sbordone, while the second result is an extension of a classical result of Martio where $\gamma=\mathbb{T}$.
\end{abstract}

\section{Preliminaries and statement of main Results}

Let $\gamma$ be a Jordan curve and let $\mathbb{T}$ be the unit circle. A function $F$ from $\mathbb{T}$ into $\gamma$ is called a sense-preserving quasi-homeomorphism from $F$ if it is the pointwise limit of a sequences of sense-preserving homeomorphisms from $\mathbb{T}$ onto $\gamma$ (for this definition we refer to [3]).

Let $\omega:[0, l] \rightarrow \mathbb{R}$ be a positive nondecreasing continuous function with $\omega(0)=0$. We will say that $\omega$ is Dini continuous if it satisfies the condition

$$
\int_{0}^{l} \frac{\omega(t)}{t} d t<\infty
$$

For $t>l$ we define $\omega(t)=\omega(l)$. A smooth Jordan curve $\gamma$ with length $l=|\gamma|$ is said to be Dini smooth if the derivative of its arc length parametrization $g$ has the modulus of continuity $\omega$ which is Dini continuous. Observe that every smooth $C^{1, \alpha}$ Jordan curve is Dini smooth.

A function $w$ is called harmonic in a region $D$ if it has the form $w=u+i v$, where $u$ and $v$ are real-valued harmonic functions in $D$.

Let

$$
P(r, t)=\frac{1-r^{2}}{2 \pi\left(1-2 r \cos t+r^{2}\right)}
$$

Received by the editors December 1, 2009 and, in revised form, December 16, 2009 and June 19, 2010 .

2010 Mathematics Subject Classification. Primary 31A05.

Key words and phrases. Harmonic extension, arc length parametrization, Dirichlet integral.

(C)2010 American Mathematical Society 
denote the Poisson kernel. Then every bounded harmonic function $w$ defined on the unit disc $\mathbb{U}:=\{z:|z|<1\}$ has the following representation:

$$
w(z)=P[F](z)=\int_{0}^{2 \pi} P(r, t-\tau) F\left(e^{i t}\right) d t,
$$

where $z=r e^{i \tau}$ and $F$ is a bounded measurable function defined on the unit circle $\mathbb{T}=\{z:|z|=1\}$

The Hilbert transform of a function $\chi$ is defined by the formula

$$
H(\chi)(\varphi)=-\frac{1}{\pi} \int_{0+}^{\pi} \frac{\chi(\varphi+t)-\chi(\varphi-t)}{2 \tan (t / 2)} d t
$$

for a.e. $\varphi$ and $\chi \in L^{1}(\mathbb{T})$. The facts concerning the Hilbert transform can be found in (11], Chapter VII).

Assume that $w=P[F](z)$ is a harmonic function defined on the unit disk $\mathbb{U}$ such that $F^{\prime} \in L^{1}(\mathbb{T})$. Then it follows that

$$
w_{\tau}=P\left[F^{\prime}\right] \text { and } r w_{r}=P\left[H\left(F^{\prime}\right)\right], \quad z=r e^{i \tau},
$$

if $w_{\tau}$ and $r w_{r}$ are bounded harmonic, because $r w_{r}$ is the harmonic conjugate of $w_{\tau}$. Here and in the remainder of this paper we use the convention $F(t)=F\left(e^{i t}\right)$ and $F^{\prime}(t)=\frac{\partial F}{\partial t}\left(e^{i t}\right)$.

The harmonic Hardy space $h^{p}$ is defined as the space of (complex-valued) harmonic functions $w$ on $\mathbb{U}$ such that

$$
\|w\|_{h^{p}}:=\sup _{0 \leq r<1}\left(\int_{0}^{2 \pi}\left|w\left(r e^{i t}\right)\right|^{p} \frac{d t}{2 \pi}\right)^{1 / p}<\infty .
$$

If $w \in h^{p}$, then by [2, Theorem 6.13] the radial limit

$$
F\left(e^{i t}\right)=\lim _{r \rightarrow 1} w\left(r e^{i t}\right)
$$

exists for almost every $e^{i t}$ in $\mathbb{T}$ and the boundary function $F\left(e^{i t}\right)$ is integrable on $\mathbb{T}$. It is well known that

$$
\|w\|_{h^{p}}^{p}=\lim _{r \rightarrow 1} \int_{0}^{2 \pi}\left|w\left(r e^{i t}\right)\right|^{p} \frac{d t}{2 \pi}=\int_{0}^{2 \pi}\left|F\left(e^{i t}\right)\right|^{p} \frac{d t}{2 \pi},
$$

which implies the formula

$$
\|w\|_{h^{p}}=\|F\|_{p}
$$

Let $w(x, y)=u(x, y)+i v(x, y)$, and let

$$
D(w)=\left(\begin{array}{ll}
u_{x} & u_{y} \\
v_{x} & v_{y}
\end{array}\right)
$$

be its Jacobian matrix at $z=x+i y$. By

$$
\|D(w)\|=\sqrt{\frac{1}{2} \operatorname{Tr}\left(D(w)^{T} D(w)\right)}
$$

we denote the mean Hilbert-Schmidt norm of $D(w)$. By using (1.3), for $1 \leq p<\infty$, we have $\|D(w)\|_{h^{p}}<\infty$ if and only if $F^{\prime}, H\left[F^{\prime}\right] \in L^{p}$. It can be shown that under the conditions $F^{\prime}, H\left[F^{\prime}\right] \in L^{p}$, it follows that $D(w) \in L^{p}(\mathbb{U})$ assuming that $p>1$.

Let us observe the case $p=\infty$. We have to find out under which condition on the boundary data $F: \mathbb{T} \rightarrow \gamma$ the harmonic mapping $w$ with $\left.w\right|_{\mathbb{T}}=F$ has bounded derivative in $\mathbb{U}$. The answer is $\|D(w)\|_{\infty}<\infty$ if and only if $F$ is continuous, 
$F^{\prime} \in L^{\infty}$ and $H\left(F^{\prime}\right) \in L^{\infty}$, where $H$ is the Hilbert transform of $F^{\prime}$. This follows from (1.3).

The fact that $F^{\prime} \in L^{\infty}(\mathbb{T})$ does not imply that $H\left(F^{\prime}\right) \in L^{\infty}(\mathbb{T})$; nor does the fact that $F^{\prime}$ is continuous imply that $H\left(F^{\prime}\right)$ is continuous.

Under the condition $F^{\prime} \in L^{\infty}(\mathbb{T})$ we have the following result, which is an extension of a corresponding theorem of Martio [8, where is treated the special case $\gamma=\mathbb{T}$.

Theorem 1.1. Let $\gamma$ be a Dini smooth Jordan curve. If $F$ is a q-Lipschitz continuous function of $\mathbb{T}$ onto $\gamma$, i.e. $F^{\prime} \in L^{\infty}(\mathbb{T})$, with $\left\|F^{\prime}\right\|_{\infty}=q$, and $w=P[F]$, then there exists a constant $C=C(q, \gamma)$ such that

$$
\left|J_{w}(z)\right| \leq C(q, \gamma),
$$

where $J_{w}:=\operatorname{det} D(w)$.

Concerning the $L^{p}$-norm of the gradient of a harmonic extension of a homeomorphism $F: \mathbb{T} \rightarrow \gamma$ of the unit circle $\mathbb{T}$ to the disk $\mathbb{U}$, Verchota in 10 showed that the harmonic mapping $w=P[F]$ has a gradient in $L^{p}$ for all $1 \leq p<2$, provided that $\gamma$ bounds a convex or a starlike domain. Note that, by a classical theorem of Rado, Choquet and Kneser, this extension is necessarily a diffeomorphism if $\gamma$ bounds a convex domain; see Chapter 3 of [4. Recently Iwaniec, Martin and Sbordone in [7. obtained a very elegant proof of this result for the case $\gamma=\mathbb{T}$, providing quite explicit constants for the norms. These problems are interesting especially in light of the recent discovery of their connection with the problem of minimizing the mean distortion of extensions of homeomorphisms of the circle; see [1].

In this paper we extend these results to the weak homeomorphisms between the unit disk and rectifiable Jordan curves as follows:

Theorem 1.2. Let $F: \mathbb{T} \rightarrow \gamma$ be a weak sense-preserving quasi-homeomorphism of the unit circle $\mathbb{T}$ onto the rectifiable Jordan curve $\gamma$ with the length $|\gamma|$. Let $w=P[F]$. Assume that $\Phi:[0, \infty) \rightarrow[0, \infty)$ is a convex increasing function. Then

$$
\int_{\mathbb{U}} \Phi\left(\frac{|2 \pi|}{|\gamma|}\|D(w)\|\right) \leq \int_{\mathbb{U}} \Phi\left(\frac{\sqrt{2}}{|1-z|}\right),
$$

where $\|D(w)\|$ is the Hilbert-Schmidt norm given by

$$
\|D(w)\|=\left(\left|w_{z}\right|^{2}+\left|w_{\bar{z}}\right|^{2}\right)^{1 / 2} .
$$

The inequality (1.5) makes sense only in the case when the integral on the righthand side converges. It converges if for example $\Phi(t)=t^{p}$ for $p<2$. The case when $\gamma$ is the unit circle is treated in [7]. However, although our method is similar, our proof gives a better inequality even for the unit circle, due to some nonsharp estimations in [7].

By using Theorem 1.2 we obtain the following isoperimetric type inequality:

Corollary 1.3. Let $F: \mathbb{T} \rightarrow \gamma$ be a weak sense-preserving quasi-homeomorphism of the unit circle $\mathbb{T}$ onto the rectifiable Jordan curve $\gamma$ with the length $|\gamma|$. Let $1 \leq p<2$ and let $w=P[F]$. Then

$$
\int_{\mathbb{U}}\|D(w)\|^{p} d x d y \leq\left(\frac{|\gamma|}{2 \pi}\right)^{p} \frac{2^{2-p / 2} \sqrt{\pi}}{2-p} \frac{\Gamma(3 / 2-p / 2)}{\Gamma(2-p / 2)} .
$$


In particular for $p=1$,

$$
\int_{\mathbb{U}}\|D(w)\| d x d y \leq \frac{2 \sqrt{2}|\gamma|}{\pi} .
$$

Furthermore for all $p \in[1,2)$,

$$
\int_{\mathbb{U}}\|D(w)\|^{p} d x d y \leq\left(\frac{|\gamma|}{4 \sqrt{2}}\right)^{p} \frac{16}{\pi(2-p)} .
$$

Remark 1.4. By (1.7) we find $\int_{\mathbb{U}} \frac{\|D(w)\|}{|\gamma|} d x d y \leq \frac{2 \sqrt{2}}{\pi} \approx 0.9$, while Example 2.3 below shows that the best constant is greater than or equal to 0.824658 . It would be of interest to find the sharp constant $c \in[0.824658,0.9]$ in this context. The Dirichlet integral $\int_{\mathbb{U}}\|D(w)\|^{2} d x d y$ can diverge, and therefore the case $p=2$ is excluded; see [10] and also our Example 2.3 below. However, if $w$ is conformal, or more generally, if $w$ is quasiconformal harmonic, then we have a similar inequality for $p=2$; see Lemma 2.2 below. If $\gamma$ is smooth enough and $w$ is quasiconformal harmonic, then we obtain the global boundedness of the Hilbert-Schmidt norm $\|D(w)\|$ in $\mathbb{U}$; see [5] and [6] and the references therein for recent developments of the theory of quasiconformal harmonic mappings.

In [1] for $\gamma=\mathbb{T}$ the inequality

$$
\int_{\mathbb{U}}\|D(w)\|^{p} d x d y \leq \frac{16 \sqrt{\pi}}{2-p}
$$

is obtained. For this special case, our inequality reduces to the better inequality

$$
\int_{\mathbb{U}}\|D(w)\|^{p} d x d y \leq\left(\frac{\pi}{2 \sqrt{2}}\right)^{p} \frac{16}{\pi(2-p)} .
$$

\section{Proofs}

Proof of Theorem 1.1. Let $g$ be an arc length parametrization of $\gamma$ with $g(0)=$ $F(0)$. Assume that $|\gamma|=l$. Since $F$ is Lipschitz, there exists a Lipschitz function $f:[0,2 \pi] \rightarrow \mathbb{R}$ such that $F(t)=g(f(t))$. Namely $f(t)=g^{-1}(F(t))$. Then

$$
F^{\prime}(t)=g^{\prime}(f(t)) \cdot f^{\prime}(t) .
$$

Since $\left|g^{\prime}(s)\right|=1$, there exists a continuous function $\theta:[0, l] \rightarrow \mathbb{R}$ such that $\theta(0)=$ $\theta(l)$ and $g^{\prime}(s)=e^{i \theta(s)}$. Thus

$$
F^{\prime}(t)=e^{i \theta(f(t))} f^{\prime}(x)
$$

From formula (1.2), for an absolutely continuous function $F$, by integration by parts we get for every $z=r e^{i \varphi} \in D$,

$$
\partial_{\varphi} w\left(r e^{i \varphi}\right)=\int_{0}^{2 \pi} F^{\prime}(x) P(r, \varphi-x) d x
$$

and

$$
\partial_{r} w\left(r e^{i \varphi}\right)=\frac{-2}{1-r^{2}} \int_{0}^{2 \pi} F^{\prime}(x) \sin (x-\varphi) P(r, \varphi-x) d x
$$


From (2.2), 2.3) and (2.1), after some elementary transformations, we obtain

$$
\begin{aligned}
J_{w}(z) & =\frac{1}{r} \operatorname{Im}\left(\overline{\partial_{r} w\left(r e^{i \varphi}\right)} \partial_{\varphi} w\left(r e^{i \varphi}\right)\right) \\
& =\frac{1}{r} \frac{-2}{1-r^{2}} \int_{0}^{2 \pi} \int_{0}^{2 \pi} \operatorname{Im}\left(\overline{F^{\prime}(x)} F^{\prime}(y)\right) \sin (x-\varphi) P(r, x) P(r, y) d x d y \\
& =\frac{1}{r} \frac{-2}{1-r^{2}} \int_{0}^{2 \pi} \int_{0}^{2 \pi} \mathcal{H}(x, y) \sin (x-\varphi) P(r, x) P(r, y) d x d y,
\end{aligned}
$$

where

$$
\mathcal{H}(x, y)=f^{\prime}(x) f^{\prime}(y) \sin [\theta(f(x))-\theta(f(y))]
$$

Furthermore

$$
|\mathcal{H}(x, y)| \leq q^{2}\left(\omega_{\gamma}(q|x-y|)\right)
$$

where $\omega_{\gamma}$ is the modulus of continuity of $g^{\prime}$ :

$$
\omega_{\gamma}(\tau):=\max \left\{\left|g^{\prime}(t)-g^{\prime}(s)\right|:|t-s| \leq \tau\right\} .
$$

Assume without loss of generality that $\varphi=0$. It follows that

$$
\left|J_{w}(z)\right| \leq q^{2} \frac{2}{r\left(1-r^{2}\right)} \int_{-\pi}^{\pi} \int_{-\pi}^{\pi} \omega_{\gamma}(q|x-y|)|\sin x| P(r, x) P(r, y) d x d y .
$$

Therefore, since $|x-y| \leq|x|$ for $|y| \leq 2|x|$ and $|x-y| \leq|y|$ for $|y| \geq 2|x|$, by taking

$$
A_{x}=\left[-2 \min \left\{|x|, \frac{\pi}{2}\right\}, 2 \min \left\{|x|, \frac{\pi}{2}\right\}\right]
$$

we have

$$
\begin{aligned}
& \int_{-\pi}^{\pi} \int_{-\pi}^{\pi} \omega_{\gamma}(q|x-y|)|\sin x| P(r, x) P(r, y) d x d y \\
& \leq \int_{-\pi}^{\pi} \int_{[-\pi, \pi] \backslash A_{x}} \omega_{\gamma}(q|y|)|\sin x| P(r, x) P(r, y) d x d y \\
&+\int_{-\pi}^{\pi} \int_{A_{x}} \omega_{\gamma}(q|x|)|\sin x| P(r, x) P(r, y) d x d y \\
& \leq \int_{-\pi}^{\pi} \omega_{\gamma}(q|y|) P(r, y) d y \int_{-|y| / 2}^{|y| / 2}|\sin x| P(r, x) d x \\
&+\int_{-\pi}^{\pi} \omega_{\gamma}(q|x|)|\sin x| P(r, x) d x \int_{A_{x}} P(r, y) d y \\
&= \frac{1-r^{2}}{2 \pi r} \int_{-\pi}^{\pi} \omega_{\gamma}(q|y|) P(r, y) \log \left(1+\frac{r \sin ^{2} y}{(1-r)^{2}}\right) d y \\
&+\frac{2}{\pi} \int_{-\pi}^{\pi} \omega_{\gamma}(q|x|)|\sin x| P(r, x) \operatorname{arccot} \frac{(-1+r) \cot \left(\min \left\{\frac{\pi}{2},|x|\right\}\right)}{1+r} d x \\
& \leq \int_{-\pi}^{\pi} \frac{\omega_{\gamma}(q|x|)}{|x|} \mathcal{K}(r, x) d x
\end{aligned}
$$

where

$$
\begin{aligned}
\mathcal{K}(r, x)= & \frac{|x| P(r, x)}{\pi}\left(\frac{1-r^{2}}{2 r} \log \left(1+\frac{r \sin ^{2} x}{(1-r)^{2}}\right)\right. \\
& \left.+2\left|\sin x \operatorname{arccot} \frac{(-1+r) \cot \left(\min \left\{\frac{\pi}{2},|x|\right\}\right)}{1+r}\right|\right) .
\end{aligned}
$$


We used the fact that

$$
y \in[-\pi, \pi] \backslash A_{x} \Leftrightarrow x \in[-|y| / 2,|y| / 2] .
$$

Further,

$$
\begin{aligned}
\frac{2}{\left(1-r^{2}\right)} \mathcal{K}(r, x)= & \frac{|x|}{2 \pi^{2}\left(1+r^{2}-2 r \cos x\right)}\left(\frac{1-r^{2}}{r} \log \left(1+\frac{r \sin ^{2} x}{(1-r)^{2}}\right)\right. \\
& \left.+4\left|\sin x \operatorname{arccot} \frac{(-1+r) \cot \left(\min \left\{\frac{\pi}{2},|x|\right\}\right)}{1+r}\right|\right) .
\end{aligned}
$$

Let us show that the function $\frac{2}{\left(1-r^{2}\right)} \mathcal{K}(r, x)$ is bounded by an absolute constant $C$.

First of all for $|x| \leq \pi$ we have $|x| \leq \pi \sin \frac{|x|}{2}$, and therefore

$$
\frac{(1-r)|x|}{2 \pi^{2}\left(1+r^{2}-2 r \cos x\right)}=\frac{(1-r)|x|}{2 \pi^{2}\left((1-r)^{2}+r\left(2 \sin \frac{x}{2}\right)^{2}\right)} \leq \frac{1}{2 \pi} \frac{\nu}{1+r \nu^{2}}
$$

and

$$
\frac{4|x| \sin x}{2 \pi^{2}\left(1+r^{2}-2 r \cos x\right)}=\frac{4|x| \sin \frac{x}{2} \cos \frac{x}{2}}{\pi^{2}\left((1-r)^{2}+r\left(2 \sin \frac{x}{2}\right)^{2}\right)} \leq \frac{4}{\pi} \frac{\nu^{2}}{1+r \nu^{2}}
$$

where

$$
\nu=\frac{2 \sin \frac{x}{2}}{1-r}
$$

Thus

$$
\frac{2}{\left(1-r^{2}\right)} \mathcal{K}(r, x) \leq \frac{1}{2 \pi} \frac{\nu}{1+r \nu^{2}} \frac{1+r}{r} \log \left(1+r \nu^{2}\right)+\frac{4 \nu^{2}}{1+r \nu^{2}} .
$$

If for example $r>1 / 2$, then from (2.5) it follows that

$$
\frac{2}{\left(1-r^{2}\right)} \mathcal{K}(r, x) \leq \frac{3}{\pi} \frac{\nu}{2+\nu^{2}} \log \left(1+\frac{1}{2} \nu^{2}\right)+\frac{8 \nu^{2}}{2+\nu^{2}}<\frac{10 \nu^{2}}{2+\nu^{2}}<10 .
$$

If $r \leq 1 / 2$, then by using the inequalities $\nu \leq \frac{2}{1-r}$ and $\log \left(1+r \nu^{2}\right) \leq r \nu^{2}$, we obtain a similar inequality for $\frac{2}{\left(1-r^{2}\right)} \mathcal{K}(r, x)$. It follows that

$$
\left|J_{w}(z)\right|<2 C \frac{q^{2}}{r} \int_{0}^{\pi} \frac{\omega_{\gamma}(q t)}{t} d t .
$$

Since $\mathbb{U}$ is simply connected, then there are two analytic functions $k$ and $h$ defined on $\mathbb{U}$ such that $w$ has the representation $w=h+\bar{k}$. Then

$$
h^{\prime}(z)=\frac{1}{2 \pi i} \int_{0}^{2 \pi} \frac{F^{\prime}(t)}{e^{i t}-z} d t
$$

and

$$
k^{\prime}(z)=\frac{1}{2 \pi i} \int_{0}^{2 \pi} \frac{\overline{F^{\prime}(t)}}{e^{i t}-z} d t .
$$

From (2.7), (2.8) and $\left|J_{w}(z)\right|=\left.|| h^{\prime}\right|^{2}-\left|k^{\prime}\right|^{2} \mid \leq \max \left\{\left|h^{\prime}\right|^{2},\left|k^{\prime}\right|^{2}\right\}$ we obtain

$$
\left|J_{w}(z)\right| \leq \frac{q^{2}}{(1-r)^{2}}, \quad r=|z| .
$$

On the other hand, since $\omega_{\gamma}(s)=\omega_{\gamma}(l)$ for $s>l$ we obtain

$$
\int_{0}^{\pi} \frac{\omega_{\gamma}(q t)}{t} d t=\omega_{\gamma}(l) \log q+\int_{0}^{\pi} \frac{\omega_{\gamma}(t)}{t} d t=\omega_{\gamma}(l) \log q+c_{\gamma}
$$


From (2.6), (2.9) and (2.10) we obtain the desired estimation.

Proof of Theorem 1.2. As in the proof of Theorem 1.1, let $g$ be an arc length parametrization of $\gamma$ and let $h$ and $k$ be two analytic functions defined in $\mathbb{U}$ such that $w=h+\bar{k}$. Since $F$ is a quasi-homeomorphism, there exists a nondecreasing function $f:[0,2 \pi] \rightarrow[0, l]$ such that $F(t)=g(f(t))$. Then

$$
F^{\prime}(t)=g^{\prime}(f(t)) \cdot f^{\prime}(t) \text { with } f^{\prime}(t) \geq 0 \text { a.e. }
$$

Assume first that $f$ is smooth. Thus $f^{\prime}$ defines the probability measure

$$
d \mu(t)=\frac{1}{|\gamma|} f^{\prime}(t) d t
$$

because

$$
\int_{0}^{2 \pi} \frac{1}{|\gamma|} f^{\prime}(t) d t=\frac{f(2 \pi)-f(0)}{|\gamma|}=1
$$

By (2.7) and (2.8) we obtain that

$$
h^{\prime}(z)=\frac{|\gamma|}{2 \pi i} \int_{0}^{2 \pi} \frac{g^{\prime}(f(t))}{e^{i t}-z} d \mu(t)
$$

and

$$
k^{\prime}(z)=\frac{|\gamma|}{2 \pi i} \int_{0}^{2 \pi} \frac{\overline{g^{\prime}(f(t))}}{e^{i t}-z} d \mu(t)
$$

Thus

$$
\frac{2 \pi}{|\gamma|} h^{\prime}(z)=-i \int_{0}^{2 \pi} \frac{g^{\prime}(f(t))}{e^{i t}-z} d \mu(t)
$$

By using the Jensen inequality we obtain that

$$
\begin{aligned}
\Phi\left(\frac{2 \pi}{|\gamma|}\left|h^{\prime}(z)\right|\right) & \leq \int_{0}^{2 \pi} \Phi\left(\left|\frac{g^{\prime}(f(t))}{e^{i t}-z}\right|\right) d \mu(t) \\
& =\int_{0}^{2 \pi} \Phi\left(\frac{1}{\left|e^{i t}-z\right|}\right) d \mu(t) .
\end{aligned}
$$

Integrating the previous inequality over the unit disk we obtain that

$$
\int_{\mathbb{U}} \Phi\left(\frac{2 \pi}{|\gamma|}\left|h^{\prime}(z)\right|\right) \leq \int_{\mathbb{U}} \int_{0}^{2 \pi} \Phi\left(\frac{1}{\left|e^{i t}-z\right|}\right) d \mu(t) .
$$

By applying Fubini's theorem we have that

$$
\int_{\mathbb{U}} \int_{0}^{2 \pi} \Phi\left(\frac{1}{\left|e^{i t}-z\right|}\right) d \mu(t)=\int_{0}^{2 \pi}\left[\int_{\mathbb{U}} \Phi\left(\frac{1}{\left|e^{i t}-z\right|}\right)\right] d \mu(t) .
$$

Introducing the change of variables $w=z e^{-i t}$ it follows that

$$
\int_{\mathbb{U}} \Phi\left(\frac{1}{\left|e^{i t}-z\right|}\right)=\int_{\mathbb{U}} \Phi\left(\frac{1}{|1-z|}\right)
$$

Therefore by (2.13) we arrive at the inequality

$$
\int_{\mathbb{U}} \Phi\left(\frac{2 \pi}{|\gamma|}\left|h^{\prime}(z)\right|\right) \leq \int_{0}^{2 \pi}\left[\int_{\mathbb{U}} \Phi\left(\frac{1}{|1-z|}\right)\right] d \mu(t)=\int_{\mathbb{U}} \Phi\left(\frac{1}{|1-z|}\right) .
$$

As $\|D(w)\|=\sqrt{\left|h^{\prime}\right|^{2}+\left|k^{\prime}\right|^{2}}$, by using (2.11) and (2.12) we obtain

$$
\frac{2 \pi}{|\gamma|}\|D(w)\| \leq \int_{0}^{2 \pi} \frac{\sqrt{2}}{\left|z-e^{i t}\right|} d t
$$


By using (2.16) and the preceding previous calculations we obtain the inequality (1.5).

When $f$ is not smooth, then as in [10, p. 893], it can be mollified by using smooth and symmetric mollifiers $f_{n}$ so that monotonicity and periodicity are retained. Then the functions $w$ and $D(w)$ are pointwise limits of the sequences $w_{n}:=P\left[g\left(f_{n}\right)\right]$ and $D\left(w_{n}\right)$, respectively. The conclusion follows by using Fatou's lemma.

Proof of Corollary 1.3. We have to solve the integral

$$
I=\int_{\mathbb{U}} \Phi\left(\frac{\sqrt{2}}{|1-z|}\right)
$$

for the convex function $\Phi(s)=s^{p}$, where $p \in[1,2)$.

Let us introduce the change of variables $z=1+r e^{i t}$. We obtain that $z \in \mathbb{U}$ if and only if $t \in[-\pi / 2, \pi / 2]$ and $r \leq 2 \cos t$. The Jacobian of the change of variables is $r$. Therefore

$$
\begin{aligned}
I & =2^{p / 2} \int_{-\pi / 2}^{\pi / 2} \int_{0}^{2 \cos t} r^{1-p} d r d t=\frac{2^{p / 2}}{2-p} \int_{-\pi / 2}^{\pi / 2}(2 \cos t)^{2-p} d t \\
& =\frac{2^{2-p / 2}}{2-p} \int_{-\pi / 2}^{\pi / 2}(\cos t)^{2-p} d t=\frac{2^{2-p / 2} \sqrt{\pi}}{2-p} \frac{\Gamma(3 / 2-p / 2)}{\Gamma(2-p / 2)} .
\end{aligned}
$$

This yields (1.6) and consequently (1.7). To prove (1.8) we estimate the quantity

$$
I:=\int_{-\pi / 2}^{\pi / 2}(\sqrt{2} \cos t)^{2-p} d t=2^{1-p / 2} \int_{-\pi / 2}^{\pi / 2}(\cos t)^{2-p} d t .
$$

Take the convex function $h(s)=s^{\frac{1}{2-p}}$ and apply the Jensen inequality to $I / \pi$. We obtain

$$
\begin{aligned}
\left(\int_{-\pi / 2}^{\pi / 2}(\sqrt{2} \cos t)^{2-p} \frac{d t}{\pi}\right)^{1 /(2-p)} & \leq \int_{-\pi / 2}^{\pi / 2}\left((\sqrt{2} \cos t)^{2-p}\right)^{1 /(2-p)} \frac{d t}{\pi} \\
& =\int_{-\pi / 2}^{\pi / 2} \sqrt{2} \cos t \frac{d t}{\pi}=\frac{2 \sqrt{2}}{\pi}
\end{aligned}
$$

It follows that

$$
2^{1-p / 2} \int_{-\pi / 2}^{\pi / 2}(\cos t)^{2-p} d t \leq \pi\left(\frac{2 \sqrt{2}}{\pi}\right)^{2-p} .
$$

This together with (1.6) implies (1.8).

Remark 2.1. The following lemma and Example 2.3 suggest that the inequality (1.7) could be sharp.

Lemma 2.2. Let $0 \leq \kappa<1$ and let $w$ be a $\kappa$-quasiconformal harmonic mapping of the unit disk onto the Jordan domain $\Omega$ bounded by the rectifiable Jordan curve $\gamma$. Then for $1 \leq p \leq 2$ :

$$
\|D(w)\|_{p}^{p} \leq \pi \frac{\left(1+\kappa^{2}\right)^{p / 2}}{(1-\kappa)^{p}}\left(\frac{|\gamma|}{2 \pi}\right)^{p} .
$$

The inequality (2.17) is attained for $w(z)=z(\kappa=0)$. 
Proof. By the Carleman isoperimetric inequality (theorem of Strebel [9, Theorem 19.9, pp. 96-98]) and the Jensen inequality, for the quasiconfomal harmonic mapping $w=h+\bar{k}\left(\left|k^{\prime}\right| \leq \kappa\left|h^{\prime}\right|\right)$, we obtain:

$$
\begin{aligned}
\int_{\mathbb{U}}\left(\left|h^{\prime}\right|^{2}+\left|k^{\prime}\right|^{2}\right)^{p / 2} & \leq \frac{1}{4 \pi}\left(1+\kappa^{2}\right)^{p / 2}\left(\int_{\mathbb{T}}\left|h^{\prime}\right|^{p / 2}\right)^{2} \leq \pi\left(1+\kappa^{2}\right)^{p / 2}\left(\int_{\mathbb{T}} \frac{\left|h^{\prime}\right|}{2 \pi}\right)^{p} \\
& \leq \pi \frac{\left(1+\kappa^{2}\right)^{p / 2}}{(1-\kappa)^{p}}\left(\int_{\mathbb{T}} \frac{\left|h^{\prime}\right|-\left|k^{\prime}\right|}{2 \pi}\right)^{p} \leq \pi \frac{\left(1+\kappa^{2}\right)^{p / 2}}{(1-\kappa)^{p}}\left(\frac{|\gamma|}{2 \pi}\right)^{p} .
\end{aligned}
$$

Example 2.3. Let

and let

$$
w(z)=\frac{2}{\pi} \arctan \frac{2 y}{\left(1-x^{2}-y^{2}\right)}
$$

$$
w_{n}(z)=\frac{2}{\pi} \arctan \frac{2 y}{\left(1-x^{2}-y^{2}\right)}+i \frac{x}{n} .
$$

Then $w_{n}(r \mathbb{T})=\gamma_{r, n}$ is a rectifiable Jordan curve. On the other hand,

$$
\begin{aligned}
w\left[\left\{r e^{i t}:-\frac{\pi}{2} \leq t \leq \frac{\pi}{2}\right\}\right] & =w\left[\left\{r e^{i t}: \frac{\pi}{2} \leq t \leq \frac{3 \pi}{2}\right\}\right] \\
& =\left[-\frac{2}{\pi} \arctan \frac{2 r}{\left(1-r^{2}\right)}, \frac{2}{\pi} \arctan \frac{2 r}{\left(1-r^{2}\right)}\right],
\end{aligned}
$$

and the function $w$ is injective in each of the $\operatorname{arcs}\left\{r e^{i t}:-\frac{\pi}{2} \leq t \leq \frac{\pi}{2}\right\}$ and $\left\{r e^{i t}: \frac{\pi}{2} \leq t \leq \frac{3 \pi}{2}\right\}$. Therefore

$$
\left|\gamma_{r}\right|:=\lim _{n \rightarrow \infty} \int_{0}^{2 \pi}\left|\frac{\partial w_{n}}{\partial t}\left(r e^{i t}\right)\right| d t=\int_{0}^{2 \pi}\left|\frac{\partial w}{\partial t}\left(r e^{i t}\right)\right| d t=\frac{8}{\pi} \arctan \frac{2 r}{\left(1-r^{2}\right)} .
$$

Thus

$$
\lim _{n \rightarrow \infty, r \rightarrow 1}\left|\gamma_{r, n}\right|=4
$$

In this special case we have

$$
\lim _{n \rightarrow \infty, r \rightarrow 1} \frac{2 \sqrt{2}\left|\gamma_{r, n}\right|}{\pi}=3.60127
$$

while

$$
\int_{\mathbb{U}}\|D(w)\|=3.29863 ; \text { cf. inequality (1.7). }
$$

Also, it is quite easy to check that for $r<1$,

$$
\int_{r \mathbb{U}}\|D(w)\|^{2}=4 \pi \log \frac{1-r^{2}}{1+r^{2}}
$$

which shows that the assumption $p<2$ for quasi-homeomorphisms in Corollary 1.3 is essential even for real harmonic functions with boundary data of bounded variation. This has been shown in [10] for circle homeomorphisms.

\section{ACKNOWLEDGMENT}

I am thankful to the referee for providing constructive comments and for help in improving the contents of this paper. 


\section{REFERENCES}

[1] K. Astala, T. Iwaniec, G.J. Martin, J. Onninen, Extremal mappings of finite distortion. Proc. London Math. Soc. (3) 91 (2005), no. 3, 655-702. MR2180459 (2006h:30016)

[2] S. Axler, P. Bourdon and W. Ramey, Harmonic function theory, Springer-Verlag, New York, 1992. MR1184139 (93f:31001)

[3] D. Bshouty, W. Hengartner, M. Naghibi-Beidokhti, p-valent harmonic mappings with finite Blaschke dilatations. XII-th Conference on Analytic Functions (Lublin, 1998). Ann. Univ. Mariae Curie-Skłodowska Sect. A 53 (1999), 9-26. MR.1775530(2001j:30016)

[4] P. Duren, Harmonic mappings in the plane, Cambridge University Press, Cambridge, 2004. MR.2048384 (2005d:31001)

[5] D. Kalaj, Harmonic quasiconformal mappings and Lipschitz spaces, Ann. Acad. Sci. Fenn. Math. 34:2 (2009), 475-485. MR2553807 (2010j:30038)

[6] D. Kalaj, M. Pavlović, On quasiconformal self-mappings of the unit disk satisfying Poisson's equation, Trans. Amer. Math. Soc, in press.

[7] T. Iwaniec, G. Martin, C. Sbordone, $L^{p}$-integrability $\& 3$ weak type $L^{2}$-estimates for the gradient of harmonic mappings of $\mathbb{D}$. Discrete Contin. Dyn. Syst. Ser. B 11 (2009), no. 1, 145-152. MR:2461814 (2009m:31003)

[8] O. Martio, On harmonic quasiconformal mappings. Ann. Acad. Sci. Fenn. Ser. A I 425, 10 pp. (1968). MR0236382 (38:4678)

[9] K. Strebel, Quadratic differentials. Ergebnisse der Mathematik und ihrer Grenzgebiete (3), 5, Springer-Verlag, Berlin, 1984. MR743423 (86a:30072)

[10] G. Verchota, Harmonic homeomorphisms of the closed disc to itself need be in $W^{1, p}, p<2$, but not $W^{1,2}$. Proc. Amer. Math. Soc. 135 (2007), no. 3, 891-894. MR 2262887(2008a:31004)

[11] A. Zygmund, Trigonometric Series. Vol. I. Second edition, Cambridge University Press, 1959. MR0107776 (21:6498)

Faculty of Natural Sciences and Mathematics, University of Montenegro, Cetinjski Put B.B., 81000, Podgorica, Montenegro

Current address: Faculty of Natural Sciences and Mathematics, University of Montenegro, Džordža Vašingtona b.b., 81000, Podgorica, Montenegro

E-mail address: davidk@t-com.me 